\title{
Modeling of Magnetic Hyperfine Field Distribution for Spherical Nanoparticles of bcc Structure
}

\author{
T. Szumiata, M. Gzik-Szumiata, K. Brzózka, \\ M. Gawroński AND B. GórKA \\ Department of Physics, Technical University of Radom \\ Krasickiego 54, 26-600 Radom, Poland
}

\begin{abstract}
We propose a simple model for temperature evolution of magnetic hyperfine field distribution of spherical bcc Fe nanoparticles. We performed mean field approximation calculations of mean spin value in each spherical shell of nanoparticle. Considering magnetic hyperfine field values reported for iron thin films we predicted possible values of hyperfine fields in the internal and surface region of the particles as a function of temperature.
\end{abstract}

PACS numbers: 78.67.Bf, 76.80.+y

\section{Introduction}

Magnetic hyperfine field distribution (HFD) is usually determined with the Mössbauer spectrometry and gives a valuable information on surface magnetic microstructure of thin layers [1-3], nanoparticles $[4,5]$ and nanocrystalline alloys $[6,7]$. We have developed a basic model in order to predict temperature evolution of HFD corresponding to the surface of bcc Fe nanoparticles.

We have assumed that the magnetic hyperfine field (MHF) influencing Fe nucleus is proportional to the local mean spin value $\left\langle S_{z}\right\rangle(T)$ and depends on the factor $f$ related to the electronic structure of environment atoms. Within a first approach we assume that this factor is approximately a linear function of the number $i$ of the nearest neighbors of the atom. Thus MHF can be described as follows:

$$
B(i, T)=B_{0}^{\mathrm{b}} f(i)\left\langle S_{z}\right\rangle(T), \quad \text { and } \quad f(i)=f_{4}+\frac{i-4}{4}\left(1-f_{4}\right),
$$

where $B_{0}^{\mathrm{b}} \approx 33.7 \mathrm{~T}$ is a value of MHF for bulk Fe at temperature $T=0 \mathrm{~K}$. For certain reasons, the factor $f(i)$ is expressed by parameter $f_{4} \equiv f(i=4)$ determined from studies of thin films surface (for bulk: $f(i=8) \equiv 1$ ). 


\section{Mean spin evolution}

Object of our investigation was a set of spherical particles of the radius $R$ (expressed as multiple of the lattice constant $a_{0}$ ) cut from bcc crystalline structure. The particle has been divided into spherical shells one unit thick. In the range of individual shell of radius $r$, average numbers of the nearest neighbors belonged to this shell $N_{r, r}^{\mathrm{NN}}$ and to adjacent ones - i.e. $N_{r, r-1}^{\mathrm{NN}}$ and $N_{r, r+1}^{\mathrm{NN}}-$ have been counted numerically and presented in Fig. 1a.
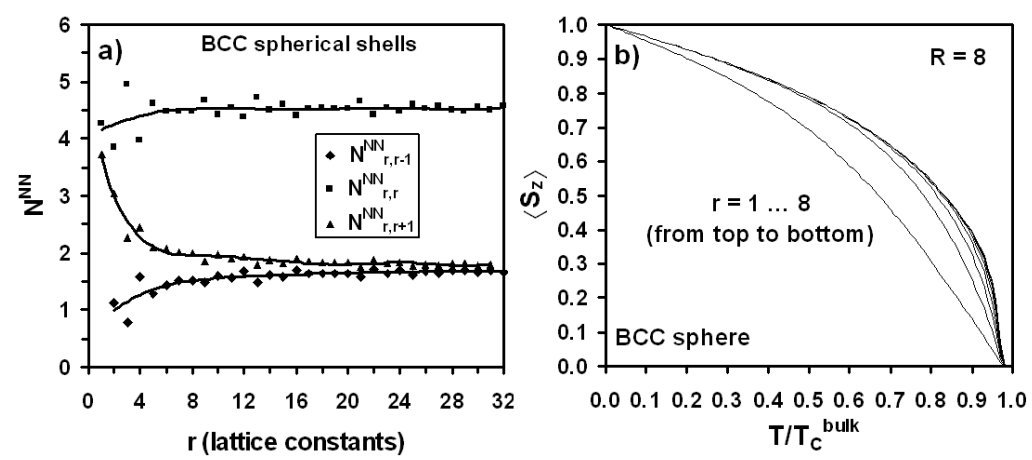

Fig. 1. Average numbers of the nearest neighbors for atoms in individual shells (a) and temperature evolution of mean spin value in each shell for particle of $R=8$ (b).

Mean field approximation (MFA) calculations of the mean spin projection have been performed within the Heisenberg model applying the method of semiclassical spin averaging [8]. In the case of isotropic particles with spins $S=1$ in zero magnetic field this approach leads to the following self-consistent system of equations for mean spin $\langle S\rangle_{r}=\left\langle S_{z}\right\rangle_{r}$ in shells of radii $r=1 \ldots R$ :

$$
\left\langle S_{z}\right\rangle_{r}=L\left(\frac{J\left(N_{r, r-1}^{\mathrm{NN}}\left\langle S_{z}\right\rangle_{r-1}+N_{r, r}^{\mathrm{NN}}\left\langle S_{z}\right\rangle_{r}+N_{r, r+1}^{\mathrm{NN}}\left\langle S_{z}\right\rangle_{r+1}\right)}{k T}\right),
$$

where $L$ is the Langevin function, $J$ - exchange integral and $k$ - the Boltzmann constant.

Results of numerical solution of the equations system (2), obtained with fixed point method, for the particle of the radius $R=8$, are displayed in Fig. 1b. As seen, in the temperature range up to the half of the Curie temperature of the bulk material the mean spin value differs significantly from that in the interior only in the eighth (surface) shell. Due to surface effects, the Curie temperature of the nanoparticle is shifted down by about $2 \%$ relatively to the bulk material. This model predicts a relatively rapid drop of the mean spin value of nanoparticle at low temperature region, in accordance with Monte Carlo results [8].

Similar MFA calculations performed for bcc [001] thin films predict values of mean spin 0.995 and 0.926 for bulk and surface region, respectively, at room temperature $\left(T=293 \mathrm{~K} \approx 0.28 T_{\mathrm{C}}\right.$, where $T_{\mathrm{C}}=1041 \mathrm{~K}$ is the Curie temperature 
for bcc bulk iron). In this case a Brillouin function instead of the Langevin one was applied in model (2), to avoid too "soft" behavior at low temperatures.

\section{Hyperfine field distribution}

Atoms located at nanoparticle surface possess different types of environments characterized by reduced coordination numbers (with respect to the bulk case) and by various values of magnetic hyperfine field. We have considered an ideal spherical surface to cut a model nanoparticle with bcc structure and we have assumed the absence of any surface reconstruction. A numerical simulation of the last spherical shell (of thickness of $1 a_{0}$ ) in the nanoparticle of the radius $R=8$ predicts five different environments corresponding to atoms possessing $8,7,6,5$ or 4 neighbors. Populations of these atoms (relatively to the total number $N=4285$ of atoms in the nanoparticle) are equal to $6.4 \%, 6.7 \%, 6.7 \%, 5.6 \%, 7.1 \%$, respectively. This numbers determine contributions of five Mössbauer subspectra (i.e. sextets in the case of ${ }^{57} \mathrm{Fe}$ spectrometry) referring to the surface shell. For such small nanoparticle a relative contribution of atoms to the surface shell is very significant $(32.5 \%)$. A dominating component (67.5\%) in the Mössbauer subspectra will be that related to a core of the nanoparticle.

In order to estimate possible values of hyperfine fields for individual sextets we used the Mössbauer data reported in [3] for thin Fe films. The sextet being characterized by the hyperfine field $(\mathrm{HF})$ of $33 \mathrm{~T}$ was attributed to bulk atomic positions (film interior, 8 nearest neighbors in bcc structure), whereas sextet of $25 \mathrm{~T}$ - to sites at atomically flat interface (4 nearest neighbors). Using these data (as well as respective values of mean spin listed in previous section) we estimated with Eqs. (1) the parameter $f_{4} \approx 0.81$. Considering results of mean spin calculations for the nanoparticle, we predicted with formula (1) the values of hyperfine field at temperatures $T=4.2 \mathrm{~K}=0.004 T_{\mathrm{C}}$ and $T=77 \mathrm{~K}=0.07 T_{\mathrm{C}}$ (Fig. 2a, b).

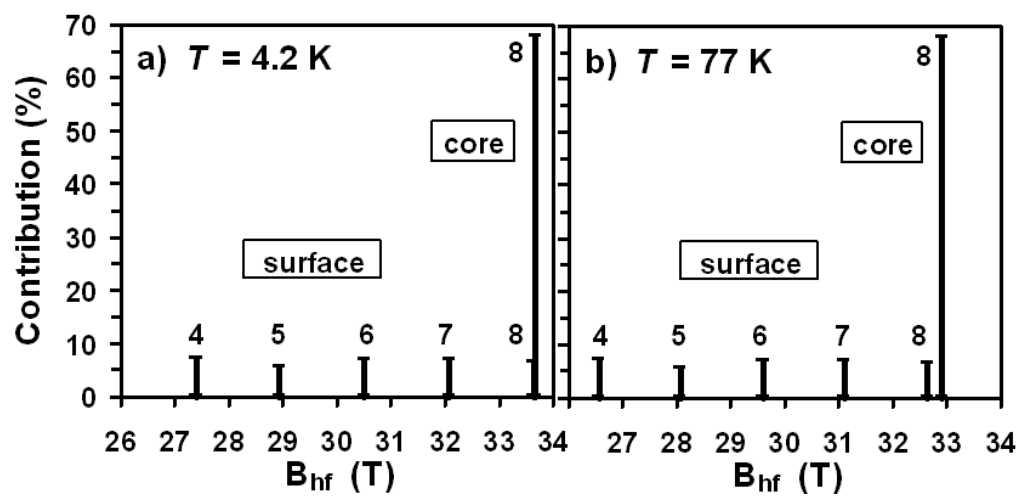

Fig. 2. HFD for Fe particle of the radius 8 at $T=4.2 \mathrm{~K}$ (a) and $T=77 \mathrm{~K}(\mathrm{~b})$. 


\section{Discussion}

Estimated values of hyperfine field for surface atoms with 8 neighbors are very similar to those for core atoms at low temperature. Our model predicts discrete HFD with four possible values of hyperfine field values corresponding to all possible environments of atoms located at surface of nanoparticle. At the realistic surface it is possible to find the atoms with smaller coordination numbers than those considered in the present work, thus one could expect a presence of lower hyperfine component in HFD. A direct comparison of our theoretical predictions with experimental data encounters difficulties due to spin relaxations effects [5], surface contamination [4], surface magnetic disorder [9], and surface strain. Thus due to these factors a real HFD of nanoparticle surface is expected to be continuous like in the case of the grains' surface in nanocrystalline alloys $[6,7]$.

\section{Conclusions}

Our model, based of MFA calculations and utilizing hyperfine parameters of thin magnetic layers, allows predicting a hyperfine field distribution for spherical nanoparticles at given temperature. We also showed that possible atomic environments (characterized by specific HF values) at the surface of bcc particle are almost equally populated. A significant role of spin relaxation effects in small particles makes it difficult to compare quantitatively the model with experimental data (from the Mössbauer spectrometry) and should be considered in the future extension of present "first-step" model.

\section{References}

[1] T. Shinjo, W. Keune, J. Magn. Magn. Mater. 200, 598 (1999).

[2] F. Klinkhammer, Ch. Sauer, E.Yu. Tsymbal, S. Handschuh, Q. Leng, W. Zinn, J. Magn. Magn. Mater. 161, 49 (1996).

[3] M. Zajạc, K. Freindl, K. Matlak, M. Ślęzak, T. Ślęzak, N. Spiridis, J. Korecki, Surf. Sci. 601, 4305 (2007).

[4] L. Theil Kuhn, A. Bojesen, L. Timmermann, K. Fauth, E. Goering, E. Johnson, M. Meedom Nielsen, S. Mørup, J. Magn. Magn. Mater. 272, 1485 (2004).

[5] N. Saegusa, M. Kusunoki, Jap. J. Appl. Phys. 29, 876 (1990).

[6] A. Ślawska-Waniewska, A. Roig, E. Molins, J.M. Grenéche, R. Żuberek, J. Appl. Phys. 81, 4652 (1997).

[7] T. Szumiata, K. Brzózka, M. Gawroński, B. Górka, J.S. Blázquez-Gámez, T. Kulik, R. Żuberek, A. Ślawska-Waniewska, J. Magn. Magn. Mater. 272, 1443 (2004).

[8] V.S. Leite, W. Figueiredo, Phys. Lett. A 359, 300 (2006).

[9] Y. Labaye, O. Crisan, L. Berger, J.M. Grenéche, J.M.D. Coey, J. Appl. Phys. 91, 8715 (2002). 